\title{
Cesariana de gestante com placenta prévia e acretismo seguido de histerectomia devido sangramento maciço
}

Cesarean section of pregnant woman with placenta previa and accretism followed by hysterectomy due to massive bleeding

Cesárea de mujer embarazada con placenta previa y acretismo seguida de histerectomía debido a sangrado masivo

Renan Cesar Loureiro ${ }^{1 *}$, Márcio Luiz Benevides ${ }^{1}$, Lucas Guimarães Carrijo ${ }^{1}$, Filipe Schwarz Godoy Ferreira ${ }^{1}$, Luís Fernando Corrêa de Souza Augusto Martins ${ }^{1}$, Ana Clara Carvalho Costa ${ }^{1}$, Feliciano Vilela Borges Ojeda ${ }^{1}$, Thamiris Pamela de Mattos ${ }^{1}$.

\section{RESUMO}

Objetivo: Relatar o caso de uma gestante multípara, com 3 cesáreas anteriores e diagnóstico de placenta prévia com acretismo submetida a cesariana seguido de histerectomia devido sangramento maciço. Detalhamento do Caso: Gestante, 36 anos, multípara, idade gestacional de 36 semanas e 5 dias, ultrassonografia evidenciando placenta percreta com suspeita de aderência vesical, sendo indicado interrupção por cesariana e histerectomia puerperal. No centro obstétrico, foram puncionados acessos venosos calibrosos e acesso venoso central. Optado por anestesia geral associado a peridural com monitorização de rotina além de pressão arterial invasiva, variação da pressão de pulso e monitor de bloqueio neuromuscular. No intraoperatorio, evolui com sangramento maciço e choque hipovolêmico, sendo necessário transfusão de 7 concentrados de hemácias, 4 plasmas fresco congelado, além do uso de droga vasoativa e fluidoterapia. Evoluiu favoravelmente com estabilidade hemodinâmica. Considerações Finais: O acretismo placentário figura entre um dos grandes desafios anestésicos e obstétricos, necessitando de um manejo cuidadoso. Aspectos como o adequado preparo do paciente, escolha do método anestésico mais seguro, bem como a otimização no tratamento são cruciais para minimizar o risco de morbimortalidade materna.

Palavras-chave: Placenta acreta, Monitorização hemodinâmica, Choque, Transfusão de componentes sanguíneos.

\begin{abstract}
Objective: Report the case of a multiparous pregnant woman, with 3 previous cesarean sections and a diagnosis of placenta previa with accretism undergoing cesarean section followed by hysterectomy due to massive bleeding. Details of the Case: Pregnant woman, 36 years old, multiparaous, gestacional age of 36 weeks and 5 days, ultrasound showing a percretal placenta with suspicion of bladder adhesion, interruption by cesarean section and puerperal hysterectomy. In the obstetric center, punctured venous access and central venous access are punctured. Opted for general anestesia associated with epidural and routine monitoring in addition to invasive blood pressure, variation in pulse pressure and neuromuscular block monitor. Intraoperatively, it develops with massive bleeding and hypovolemic shock, requiring transfusion of 7 hemaceae concentrate, 4 fresh frozen plasmas, in addition to the use os vasoactive drugs and fluid therapy. It envolved favorably with hemodynamic stability. Final Considerations: Placental accretism is one of the major anesthetic and obstetric challenges, requiring careful management. Aspects such as adequate patient preparation, choosing the safest anesthetic method, as wellas optimizing treatment are crucial to minimize the risk of maternal mobidity and mortality.
\end{abstract}

Keywords: Placenta accreta, Hemodynamic monitoring, Shock, Blood component transfusion.

\footnotetext{
${ }^{1}$ Hospital Universitário Júlio Muller (HUJM), Cuiabá - MT. *E-mail: renan_loureiro_11@hotmail.com
} 


\section{RESUMEN}

Objetivo: Reporte el caso de uma gestante multípara, con 3 cesáreas previas y diagnóstico de placenta previa con acretismo sometida a cesárea seguida de histerectomia por sangrado massivo. Detalles del Caso: Gestante de 36 años, multípara, edad gestacional de 36 semanas y 5 días, ecografia que muestra placental percretal con sospecha de adherencia vesical, interrupción por cesárea e histerectomía puerperal. En em centro de obstetricia, se perfora el acceso venoso perforado y el acceso venoso central. Se optó por anestesia general asociada a epidural y monitorización de rutina además de presión arterial invasiva, variación de la presión del pulso y monitor de bloqueio neuromuscular. Intraoperatoriamente se desarrolla con sangrado masivo y shock hipovolémico, requiriendo transfusión de 7 concentrados de glóbulos rojos, 4 plasma fresco congelado, además del uso de fármacos vasoactivos y fluidoterapia. Evolucionó favorablemente con estabilidad hemodinámica. Consideraciones Finales: El acretismo placentario es uno de los principales desafíos anestésicos y obstétricos, que requiere un tratamiento cuidadoso. Aspectos como la preparación adecuada de la paciente, la elección del método anestésico más seguro, así como la optimización del tratamiento son cruciales para minimizar el riesgo de morbimortalidad materna.

Palabras clave: Placenta accreta, Monitorización hemodinámica, Shock, Transfusión de componentes sanguíneos.

\section{INTRODUÇÃO}

A Organização Mundial de Saúde (OMS), preconiza a taxa de cesariana em 15\% de todos os partos, porém nas últimas décadas ela se tornou o modo mais comum de nascimento no país, representando a via de parto em $56 \%$ de todos os nascidos vivos (WHO, 2015). A nível mundial, o Brasil ocupa o segundo lugar no ranking de cesáreas (56\%), ficando atrás apenas da República Dominicana (57\%) (SOUZA JP, et al., 2010).

Cesariana repetida é um fator de risco para o desenvolvimento de complicações gestacionais, dentre elas a placenta prévia e acretismo placentário, destacando-se esta última (IACOVELLI A, 2020). Placenta prévia é definida pela implantação da placenta no segmento inferior do útero podendo estar parcialmente ou totalmente sobreposto ao orifício interno. Essa condição é frequentemente complicada por invasão de vilosidades placentárias causando placenta acreta ou increta. Devido a localização anormal e a invasão do tecido placentário, é provável ocorrer grave sangramento materno especialmente no terceiro trimestre da gravidez e no início do trabalho de parto (KOLLMANN M, et al., 2016).

Cirurgias uterinas, como miomectomia ou curetagem, idade materna avançada, multiparidade e tabagismo também são considerados fatores riscos de desenvolvimento de acretismo placentário. A detecção prévia dessas alterações permite um planejamento mais adequado para o perioperatório com redução no risco de perda sanguínea e morbimortalidade materna (HWU YW, 2006; GIELCHINSKY Y, et al., 2002; KONG CW e TO WWK, 2020).

Este relato de caso tem objetivo de apresentar o manejo de uma paciente multípara, com três cesarianas anteriores, com diagnóstico de placenta prévia com acretismo seguido de histerectomia devido sangramento maciço.

\section{RELATO DE CASO}

Paciente do sexo feminino, 36 anos, gestante, $85 \mathrm{~kg}$, índice de massa corpórea: $31,7 \mathrm{~kg} / \mathrm{m}^{2}$, com 3 cesarianas prévias e idade gestacional (IG) de 36 semanas e 5 dias. Na ultrassonografia (USG) foi evidenciado placenta prévia centro-total percreta com suspeita de aderência vesical, sendo indicado interrupção por cesariana com histerectomia puerperal (Figura 1). 
Figura 1 - Ultrassonografia obstétrica evidenciando acretismo placentário (seta) com suspeita de aderência vesical.

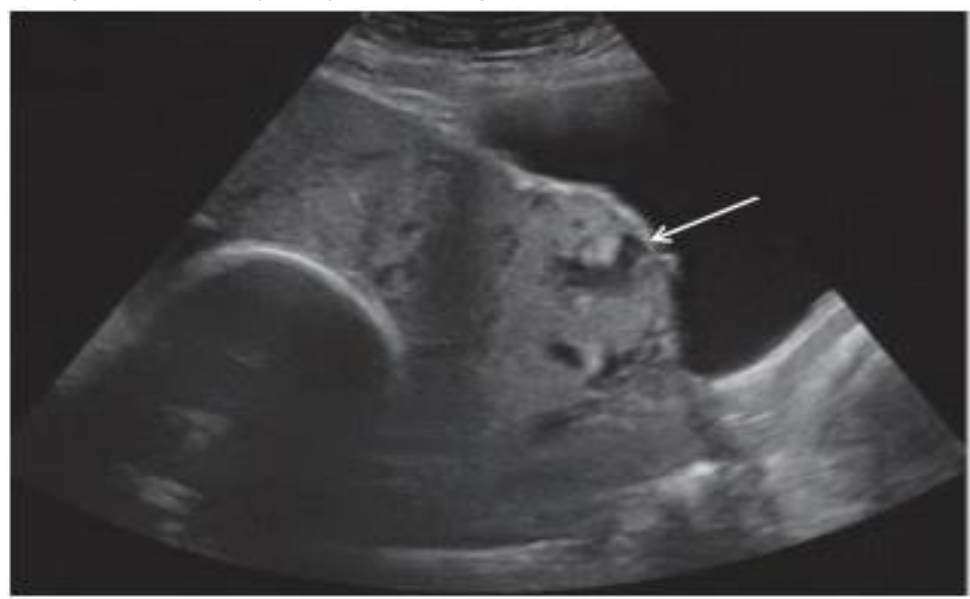

Fonte: Loureiro RC, et al., 2020.

No pré anestésico foi orientado sobre o manejo proposto e não foi detectado nenhuma comorbidade prévia ou alteração em exame físico, apresentando hemoglobina $(\mathrm{Hb}): 11.8 \mathrm{~g} / \mathrm{dL}$, hematócrito: $36.2 \%$, plaquetas: 168 mil. Previamente a entrada da paciente no centro obstétrico, concentrado de hemácias $(\mathrm{CH})$ e plasma fresco congelado (PFC) já estavam em sala, bem como solução de noradrenalina $(16 \mathrm{mcg} / \mathrm{ml})$ já preparada em bomba de infusão contínua (BIC).

Realizada monitorização com cardioscopia, oximetria de pulso, pressão arterial e monitor de bloqueio neuromuscular, seguido de 2 acessos venosos calibrosos periféricos (cateter 16G e 14G) em membros superiores, com auxílio de USG foi puncionado acesso venoso central (AVC) duplo lúmen em jugular interna direita e cateterização da artéria radial a esquerda para monitorização avançada da pressão arterial invasiva (PAl) e variação da pressão de pulso (delta PP).

Optado por bloqueio peridural pela possibilidade de analgesia pós-operatória, em decúbito lateral esquerdo, punção em L3-L4 com agulha de Tuohy 16G e técnica de Doglioti com passagem de cateter seguido de infusão de morfina $2 \mathrm{mg}+$ fentanil $100 \mathrm{mcg}$. Novamente em decúbito dorsal, elevada a cabeceira em $30^{\circ}$, iniciado pré oxigenação sob máscara facial com oxigênio a $100 \%$ por 5 minutos e infusão de remifentanil $70 \mathrm{mcg}$ + lidocaína $100 \mathrm{mg}$ + propofol $150 \mathrm{mg}$ + succinilcolina $100 \mathrm{mg}$ via endovenosa, seguido de laringoscopia com Cormack 1 e intubação orotraqueal com tubo orotraqueal número 7,0 confirmado por capnografia, manutenção da anestesia com sevoflurano $2 \%$ + remifentanil $0,2 \mathrm{mcg} / \mathrm{kg} / \mathrm{min}$ em BIC.

Após histerotomia foi visualizada invasão placentária em região de 2/3 do corpo inferior e istmo do útero. Extração do recém-nascido em apresentação pélvica ocorreu em menos de 1 minuto após indução anestésica, apresentando índice de Apgar de 7 no primeiro minuto e 9 no quinto minuto. Foi infundido 10 unidades de ocitocina após clampeamento do cordão, porém evoluiu com sangramento maciço e choque hipovolêmico, sendo decidido a realização de histerectomia puerperal com rafia da bexiga.

Houve perda estimada em 3 litros de sangue. Foi administrado de maneira imediata noradrenalina com titulação aumentada conforme necessidade $(0,05$ a $0,5 \mathrm{mcg} / \mathrm{kg} / \mathrm{min})$, transfusão de $7 \mathrm{CH}$ e 4 PFC e ressuscitação volêmica com $1500 \mathrm{ml}$ de hidroxietilamida (HEA), $2500 \mathrm{ml}$ de ringer lactato e $1000 \mathrm{ml}$ de soro fisiológico 0,9\%.

Todo o manejo hemodinâmico foi pautado em gasometrias seriadas na qual foram encontrados os valores de $\mathrm{pH}, \mathrm{BE}$ e lactato (menor e maior valor), respectivamente 7,26 e 7,38; -9,7 mmol/L e -2,4 mmol/L; 3,7 mmol/L e $1,7 \mathrm{mmol} / \mathrm{L}$. Além das gasometrias, o manejo foi guiado pela monitorização avançada da PAl e delta PP. Ao final da cirurgia, paciente apresentou estabilidade hemodinâmica, evoluindo para extubação, não havendo necessidade de terapia intensiva no pós-operatório.

Ao receber alta da sala de recuperação anestésica apresentava $\mathrm{Hb}: 7.4 \mathrm{~g} / \mathrm{dL}$. Realizado acompanhamento com visitas de rotina na enfermaria não havendo queixas de dor ou náusea e vômito, retirado o cateter peridural após 48 horas da cirurgia. Recebeu alta hospitalar no terceiro dia pós operatório. 


\section{DISCUSSÃO}

A presença de cesáreas prévias é o principal fator de risco para desenvolvimento de placenta prévia e acretismo. Em coorte prospectiva com 30.132 gestantes nos Estados Unidos da América foi detectado que em mulheres com placenta prévia, o risco de acretismo aumentou dramaticamente naquelas com cesáreas anteriores e mesmo em mulheres sem placenta prévia o aumento no número de cesárias também elevou 0 risco de placenta acreta, visto também que a morbidade materna aumentou substancialmente em todas as mulheres com placenta acreta em comparação com aquelas que não tem acretismo placentário (SILVER RM, et al., 2006).

O diagnóstico de acretismo placentário realizado de maneira prévia no pré-natal, permite a preparação para o manejo mais adequado e seguro. A USG perinatal tem uma sensibilidade de $77-93 \%$ e especificidade de $71-96 \%$ para detecção de placenta acreta. A ressonância magnética (RM) tem uma sensibilidade de 80 $88 \%$ e especificidade de $65-100 \%$ em diagnosticar acretismo, apresentando superioridade na detecção da profundidade da invasão do trofoblasto (HULL AD e RESNIK R, 2010). Devido indisponibilidade de RM em nosso serviço, foi realizado apenas USG o qual evidenciou o acretismo.

A coordenação entre as equipes é uma peça-chave para o sucesso no tratamento do acretismo placentário. Obstetras, anestesiologistas, radiologistas intervencionistas, banco de sangue e enfermagem necessitam estar bem alinhados para evitar atrasos nas condutas (FUCHS KM, et al., 2009). Foi demonstrado que um planejamento cuidadoso diminui a perda sanguínea, a necessidade de hemoderivados e a morbimortalidade perioperatória (OPHIR E, et al., 2009). Por essas razões é indicado a cesariana eletiva, de preferência acima de 34 semanas, com conhecimento prévio de toda equipe, conforme realizado.

O fluxo sanguíneo através de cada artéria uterina aumenta de 100 para $350 \mathrm{ml} /$ minuto na gravidez (THALER I, et al., 1990). Na placenta acreta, os vasos sanguíneos podem ser ainda mais calibrosos e incapazes de sofrer vasoespasmo, cursando com uma hemorragia descontrolada (ZELOP CM, et al., 1993). Rosen T, et al. (2008) demonstrou que a perda de sangue variou entre 2.5 a 5 litros, sendo necessário um volume médio de $10 \mathrm{CH}$, com choque hemorrágico ocorrendo em mais da metade dos casos e coagulação intravascular disseminada ocorrendo em $25 \%$ dos pacientes. Destaca-se que no centro obstétrico, foram puncionados 2 acessos venosos calibrosos e AVC visando a administração de drogas vasoativas, reposição de cristalóides, hemoderivados e HEA.

De maneira geral a anestesia de escolha para procedimentos obstétricos é o bloqueio do neuroeixo, que apresenta cerca de 17 vezes menos complicações que a anestesia geral, incluindo falha na intubação endotraqueal, aspiração de conteúdo gástrico, hipóxia e 1,7 vezes menos risco de morte materna (LYNCH J e SCHOLZ S, 2005). Dentre as técnicas do bloqueio de neuroeixo, a raquianestesia é a técnica mais empregada no Brasil, porém neste presente relato de caso foi optado por anestesia geral, pois um bloqueio simpático poderia acarretar importante hipotensão em paciente que se esperava grandes perdas sanguíneas.

Levando em consideração que gestante é considerada estômago cheio e pode apresentar preditores de via aérea difícil, foi optado por anestesia geral com indução em sequência rápida com manobra de Sellick com drogas de rápido início de ação, como remifentanil, lidocaína, propofol e succinilcolina. Além disso, conforme Paech M, et al. (2009); Hawkins JL, (2003), em procedimentos com alto risco de instabilidade hemodinâmica a anestesia geral pode ser mais interessante, por ser mais facilmente controlado o nível da anestesia, além de não apresentar bloqueio simpático que possa agravar o choque como na anestesia do neuroeixo.

Ademais visando oferecer analgesia pós-operatória, foi realizado bloqueio peridural analgésico com passagem de cateter peridural com administração intermitente de anestésico local e opióides para otimizar analgesia pós-operatória, visando melhorar a satisfação do paciente e acelerar sua recuperação. Além disso pode prevenir picos hipertensivos associados à dor os quais podem acarretar maior risco de sangramento.

Salienta-se a importância da utilização do lactato sérico para monitoramento da perfusão tecidual periférica. Park YJ, et al. (2018) demonstraram que lactato sérico maior que $2,6 \mathrm{mmol} / \mathrm{L}$ previu uma mortalidade hospitalar em 30 dias significativamente maior do que o grupo com baixo teor de lactato. No paciente relatado, foi realizado avaliação seriada do lactato sérico através da gasometria arterial onde no momento mais crítico apresentou valor de $3,7 \mathrm{mmol} / \mathrm{L}$ evoluindo satisfatoriamente, com as medidas adotadas, para um valor de $1,7 \mathrm{mmol} / \mathrm{L}$. 
Em procedimentos com grande perda sanguínea e risco de instabilidade hemodinâmica, o uso de monitorização avançada é importante para guiar as condutas terapêuticas. As variações cíclicas do débito cardíaco que ocorrem com a ventilação mecânica sofrem influência do volume corrente e da pressão positiva expiratória final e são mais acentuadas em pacientes hipovolêmicos. A pressão de pulso é diretamente proporcional ao volume sistólico e inversamente proporcional à elastância aórtica (ROCHA P, et al., 2014).

Como a elastância permanece constante entre um batimento e outro, a pressão de pulso pode ser utilizada como substituta para o volume sistólico, consequentemente as variações na pressão de pulso que ocorrem durante a ventilação mecânica refletem as variações no débito cardíaco. Ressalta-se as condições necessárias para o uso do delta PP, que incluem: paciente deve estar em ventilação mecânica, sedado e paralisado; ventilação deve estar em modo de controle de volume; não deve haver arritmia, shunt intracardíaco ou doença valvular significativa (ROCHA P, et al., 2014).

O delta PP representa a diferença entre a pressão de pulso máxima e a pressão de pulso mínima dividida pela média das duas ao longo de um ciclo respiratório. Sua interpretação se dá com ponto de corte de 13\% para discriminar entre respondedores (Delta PP $>13 \%$ ) e não respondedores (Delta PP $<13 \%$ ) a volume com uma sensibilidade de $94 \%$ e especificidade de $96 \%$. Adicionalmente, o Delta PP mostrou-se um indicador de resposta a infusão de volume mais confiável que a pressão venosa central e a pressão de oclusão da artéria pulmonar (ROCHA P, et al., 2014). Durante todo o procedimento relatado, a infusão de volume foi avaliada através dos valores do delta PP, realizando assim uma hidratação guiada por metas, evitando excesso de fluídos.

A embolização das artérias uterinas através da radiologia intervencionista tem mostrado resultados benéficos quanto a redução da morbimortalidade materna, reduzindo drasticamente o sangramento e a necessidade de histerectomia (SALAZAR GMM, et al., 2009). A técnica percutânea envolve a oclusão temporária das artérias ilíacas internas ou artérias uterinas utilizando-se balões de angioplastia, sendo o procedimento realizado imediatamente antes da cesariana (THON S, et al., 2010). Bouvier A, et al. (2012) demonstraram que, em uma casuística de 14 pacientes com acretismo placentário submetidas à embolização perioperatória da artéria uterina, em oito casos $(57,1 \%)$, foi possível evitar a histerectomia.

Entretanto o sucesso desse tipo de procedimento demanda a estrutura física de um serviço de hemodinâmica e um aparelho de radioscopia qualificado, assim como o apoio de uma equipe multiprofissional treinada. A ausência de algum desses fatores pode comprometer os resultados positivos dessa intervenção (BISHOP S, et al., 2011). Em nosso hospital não há serviço de hemodinâmica disponível, impossibilitando assim essa opção de tratamento.

Portanto, conclui-se que o acretismo placentário figura entre um dos grandes desafios anestésicos e obstétricos, necessitando de um manejo cuidadoso visando diagnóstico prévio do acretismo, bem como a coordenação de toda a equipe na sala operatória, preparação da paciente com monitorização adequada e acessos venosos calibrosos, segurança na escolha na anestesia a ser utilizada, insumos e drogas prontos para uso, além da otimização no tratamento visando menor risco de hemorragias e óbito.

\section{REFERÊNCIAS}

1. BETRAN AP, et al. WHO Working Group on Caesarean Section. WHO Statement on Caesarean Section Rates. BJOG. 2016;123(5):667-670.

2. BISHOP S, et al. Multiple complications following the use of prophylatic internal iliac artery balloon catheterisation in a patient with placenta percreta. Int J Obstet Anesth. 2011;20(1):70-3.

3. BOUVIER A, et al. Planned Caesaean in the inetrevntional radiology cath lab to enable immediate uterine artery embolization for the conservative treatment of placente accreta. Clin Radiol. 2012; 67:1089-1094.

4. FUCHS KM, et al. Optimizing outcomes through protocols, multidisciplinary drills, and simulation. Semin Perinatol 2009; 33:104-108.

5. GIELCHINSKY Y, et al. Placenta accreta - summary of 10 years: a survey of 310 cases. Placenta 2002; 23:210-214.

6. HAWKINS JL. Anesthesia-related maternal mortality. Clin Obstet Gynecol 2003; 46:679-687.

7. HULL AD, RESNIK R. Placenta accreta and postpartum hemorrhage. Clin Obstet Gynecol 2010; 53:228-236

8. HWU YM, et al. Parallel vertical compression sutures: a technique to control bleeding from placenta praevia or accreta during caesarean section. BJOG. 2005 Oct;112(10):1420-3.

9. IACOVELLI A, et al. Risk factors for abnormally invasive placenta: a systematic review and meta-analysis. J Matern Fetal Neonatal Med. 2020 Feb;33(3):471-481. 
10. KONG CW, TO WWK. Risk factors for severe postpartum haemorrhage during caesarean section for placenta praevia. J Obstet Gynaecol. 2020 May;40(4):479-484.

11. LYNCH J, SCHOLZ S. Anaesthetic-related complications of caesarean section. Zentralbl Gynakol 2005; 127:91-95.

12. KOLLMANN M, et al. Placenta praevia: incidence, risk factors and outcome, The Journal of Maternal-Fetal \& Neonatal Medicine 2016, 29:9, 1395-1398

13. OPHIR E, et al. Abnormal placental invasion - a novel approach to treatment: case report and review. Obstet Gynecol Surv 2009; 64:811-822.

14. PAECH M, et al. A prospective study of awareness and recall associated with general anesthesia for cesarean section. Obstetric Anesthesia Digest 2009; 29:155-156.

15. PARK YJ, et al. Serum lactate upon emergency department arrival as a predictor of 30-day in-hospital mortality in an unselected population. PLoS One. 2018 Jan 2;13(1):e0190519.

16. ROCHA P, et al. Avaliação hemodinâmica em paciente criticamente enfermo. Braz. J. Nephrol., v. 32, n. 2, p. 201212, jun. 2010.

17. ROSEN T. Placenta accreta and cesarean scar pregnancy: overlooked costs of the rising cesarean section rate. Clin Perinatol 2008; 35:519-529.

18. SALAZAR GMM, et al. Transcatheter endovascular techniques for management of obstetrical and gynecologic emergencies. Tech Vasc Intervent Radiol 2009; 12:139-147

19. SILVER RM, et al. Maternal morbidity associated with multiple repeat cesarean deliveries. Obstet Gynecol 2006; 107:1226-1232.

20. SOUZA JP, et al. Maternal near miss and maternal death in the World Health Organization's 2005 global survey on maternal and perinatal health. Bulletin of the World Health Organization. $2010 \mathrm{Feb} ; 88(2): 113-9$.

21. THALER I, et al. Changes in uterine blood flow during human pregnancy. Am J Obstet Gynecol 1990; 162:121-125.

22. THON S, et al. Prophylactic endovascular placement of internal iliac occlusion balloon catheters in parturients with placenta accreta: a retrospective case series. Int J Obstet Anesthesia 2010; 20:64-70.

23. ZELOP CM, et al. Emergency peripartum hysterectomy. Obstet Gynecol 1993; 168:1443-1448. 